\title{
Contribution of Computed Tomographyin The Diagnosis of Pulmonary Tuberculosis at Kiffa Regional Hospital, Assaba
} \author{
and Leonardo Kishi Basco ${ }^{5}$ \\ ${ }^{1}$ Department of Internal and Medicine Infectious Diseases Kiffa regional hospital, Africa \\ ${ }^{2}$ Department of Radiology, Centre National d'Oncologie, Africa \\ ${ }^{3}$ Department of Medicine Infectious Diseases, CHU Yalgado Ouédraogo, West Africa \\ ${ }^{4}$ Department of Medicine Infectious Diseases, Hôpital du Point G, West Africa \\ ${ }^{5}$ Aix Marseille Univ, France
}

Boushab Mohamed Boushab ${ }^{1 *}$, Sidi Yeslem Ould-Bahiya ${ }^{2}$, Mamoudou Savadogo $^{3}$, AbdoulayeMamadou Traoré ${ }^{4}$

Submission: August 20, 2018; Published: October 31, 2018

*Corresponding author: Boushab Mohamed Boushab, Médecine Interne et Maladies Infectieuses, Centre hospitalier de Kiffa, Assaba, Mauritania, Africa.

\section{Abstract}

Background: Computed tomography (CT) is currently the most sensitive and specific medical imaging examination for lung diseases, but it's use should be based on the results of clinical examination and chest X-ray.

Objectives: This study aimed to describe the contribution of CT in the diagnosis of pulmonary tuberculosis (TB) in symptomatic patients with normal chest X-ray.

Materials and methods: This was a prospective study conducted in 2017 in Kiffa Regional Hospital, Mauritania, in patients requiring a chest X-ray for suspected pulmonary TB. CT imaging was performed if sputum examination was negative and chest X-ray was normal.

Results: Twenty-four TB cases (mean age, 43.7 years; sex ratio M/F, 2.0) were included. The presenting symptoms included chest pain (79\%), chronic cough (71\%), fever (63\%), and dyspnea (46\%). CT imaging showed medium lobar alveolar condensation with a bilateral centrilobular and micronodular bronchogram (13\%), interstitial anomalies in the form of numerous inter- and intra-lobular pleural cross-links in the middle and lower right lobar lobes (25\%), diffuse fibrotic interstitial pneumopathy (17\%), and micronodules, confluent nodules, condensations, and unilateral cavities (42\%).

Conclusion: CT is particularly valuable for tracking and monitoring parenchymal nodules, for establishing the diagnosis and monitoring of infectious pneumonitis, secondary thoracic complications, and exploration of unexplained lung disease. Although chest radiography has several shortcomings to establish the diagnosis of TB in patients who have negative sputum smears, it is still useful for initial screening. For cases with clinical signs of active disease and normal chest X-ray, CT is indicated.

Keywords: Pulmonary tuberculosis; Radiography; Scanography; Mauritania

\section{Introduction}

Tuberculosis (TB) is a public health problem worldwide, especially in developing countries. The persistence of tuberculosis is partly related to increased longevity and aging of the population and emergence of drug-resistant Mycobacterium tuberculosisstrains [1]. In resource-limited settings where sputum culture and nucleic acid amplification techniques are not routinely available, diagnosis of pulmonary TB is based on clinical signs and symptoms (fever, productive cough, purulent sputum, hemoptysis, dyspnea, weight loss, loss of appetite), microscopic examination of sputum smear, and chest X-ray [2]. An additional difficulty is that the diagnosis can be difficult when signs and symptoms are either atypical or non-specific, such as acute pneumonia, chroniccough, fever, and diarrhea. Autopsies in several countries have shown that an accurate diagnosis of pulmonary tuberculosis was made in only about $50 \%$ of cases [15]. In many cases, diagnosis is established too late, allowing the spread of contagion from undetected cases. In countries where both tuberculosis and Human Immunodeficiency Virus (HIV)/ 
Acquired Immunodeficiency Syndrome (AIDS) are endemic, HIV testing is essential in all patients with tuberculosis. A sociological difficulty also lies in the fact that diagnostic approaches of these two diseases are historically different. Anti-tuberculosis campaigns have been public, whereas HIV/AIDS screening is generally individual and confidential in many countries. It has been reported that currently only about $10 \%$ of Africans are suffering from pulmonary tuberculosis [3].

In industrialized countries, Computed Tomography (CT) tends to replace traditional diagnostic methods not only to establish the definite diagnosis of TB, but also to monitor the evolution of the disease. Most often, the radiographic images in a standard chest X-ray are quite suggestive of pulmonary TB. However, patients in whom chest X-rays present atypical features that confound interpretation of the radiographic images would benefit from the high-resolution CT imaging. Although CT has the advantage of providing a more accurate imaging, the cost involved in the acquisition and maintenance of the equipment and patient charges may be too high, especially in less developed countries where the incidence of TB remains high. Within this context, it is essential to determine the place of CT to establish the diagnosis of pulmonary TB in Mauritania. The aim of the present study was to compare CT to standard chest radiography in establishing TB diagnosis.

\section{Materials and Methods}

This was a prospective study conducted in Kiffa Regional Hospital from January 1 to December 31, 2017, in all patients requiring a chest X-ray. Diagnosis of pulmonary TB was performed on an ambulatory basis, except in patients presenting severe signs and symptoms (hemoptysis, altered consciousness) who were hospitalized. Three sputum samples were collected from all patients with suspected pulmonary TB. Patients were included after informed consent if (i) they had been treated unsuccessfully with non-specific antibiotic therapy, (ii) had at least three negative microscopic examinations of sputum specimens, (iii) but presented X-ray and/or CT evidence of TB, and (iv) responded favorably to anti-tuberculosis therapeutic test. Patients with positive sputum smears or radiological abnormalities and treatment success with non-anti-TB antibiotics were excluded. All CT scans were reread blindly by an experienced radiologist to identify parenchymal lesions and theirbronchial, mediastinal, pleural and parietal morphology.

Patients were screened for HIV by ELISA, and the results were confirmed by Western blot. For each patient, clinical and epidemiological data (age, sex, geographical origin) and history of anti-TB treatment and evolution of the disease (i.e., the results of sputum smears after 2, 3, 5 and 6 months of treatment) were collected and recorded. The anti-TB treatment for all patients consisted of the combination regimen rifampicin $(10 \mathrm{mg} / \mathrm{kg} /$ day), isoniazid (5 mg/kg/day), ethambutol $(20 \mathrm{mg} / \mathrm{kg} /$ day), pyrazinamide $(30 \mathrm{mg} / \mathrm{kg} /$ day) for two months, followed by dual therapy with rifampicin and isoniazid for four months at the same dosages.

\section{Results}

During the period of twelve months, 24 patients who met the inclusion criteria were included. The study focused only on patients who were able to perform both chest X-ray and thoracic CT. The mean age was 43.7 years (range, 15-76 years). The sex ratio of men to women was 2.0. The majority of our patients $(20 / 24,83 \%)$ came from rural areas. The mean time to diagnosis was seven weeks, with general signs dominated by chest pain (79\%), chronic cough (71\%), fever (63\%), and dyspnea (46\%). Other presenting signs and symptoms included hepatomegaly (38\%), general impairment (25\%), polyadenopathy $(17 \%)$, and hemoptysis (13\%). Chest X-ray in these patients was normal. The following CT abnormalities were observed, in decreasing order: medium lobar alveolar condensation with a bilateral centrilobular and micronodular bronchogram (Figure 1) (3cases of 24 patients, 13\%), interstitial anomalies in the form of numerous inter- and intra-lobular pleural cross-links in the middle and lower right lobar lobes (25\%) (Figure 2), diffuse fibrotic interstitial pneumopathy (17\%) (Figure 3), and micronodules, confluent nodules, condensations, and unilateral cavities (42\%) (Figure 4). Bacteriological examination of the sputum for M. tuberculosis and HIV serology were negative in all patients.

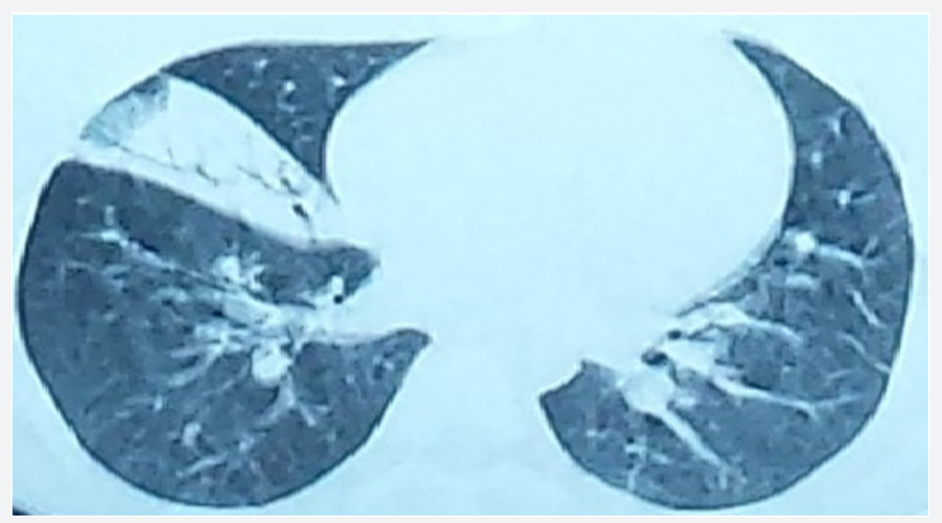

Figure 1: Bilateral lobar alveolar condensation with bronchogram and bilateral centrilobular micronodules. 


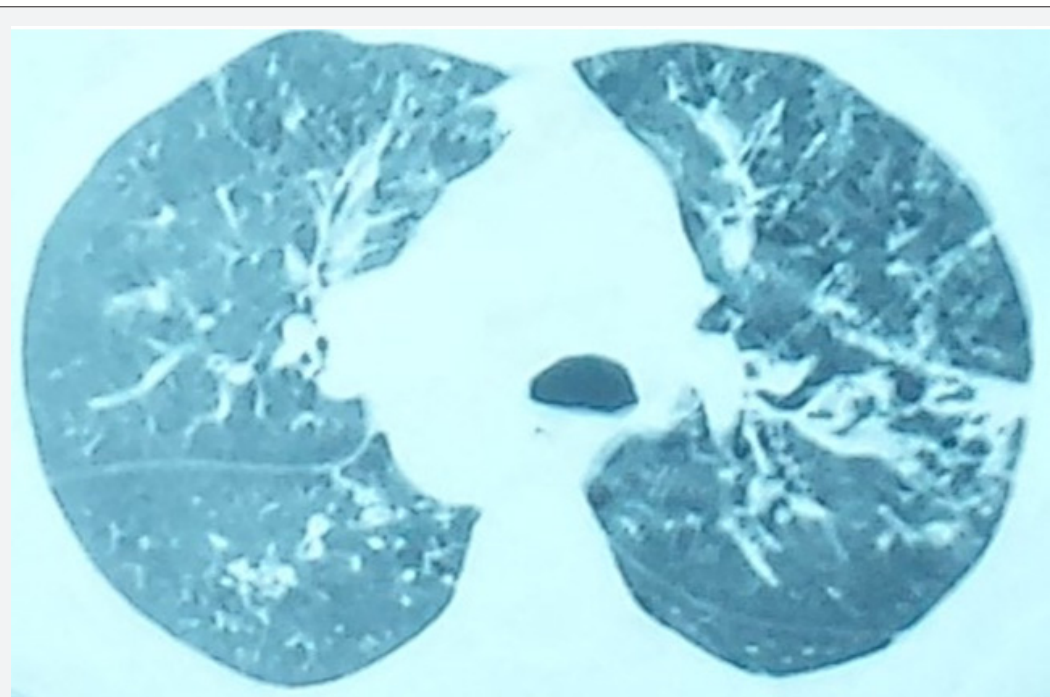

Figure 2: Diffuse interstitial pneumonitis that is not yet fibrosing to yield interstitial anomalies in the form of numerous inter-and intra-lobular pleural cross-links in the middle and lower right lobes.

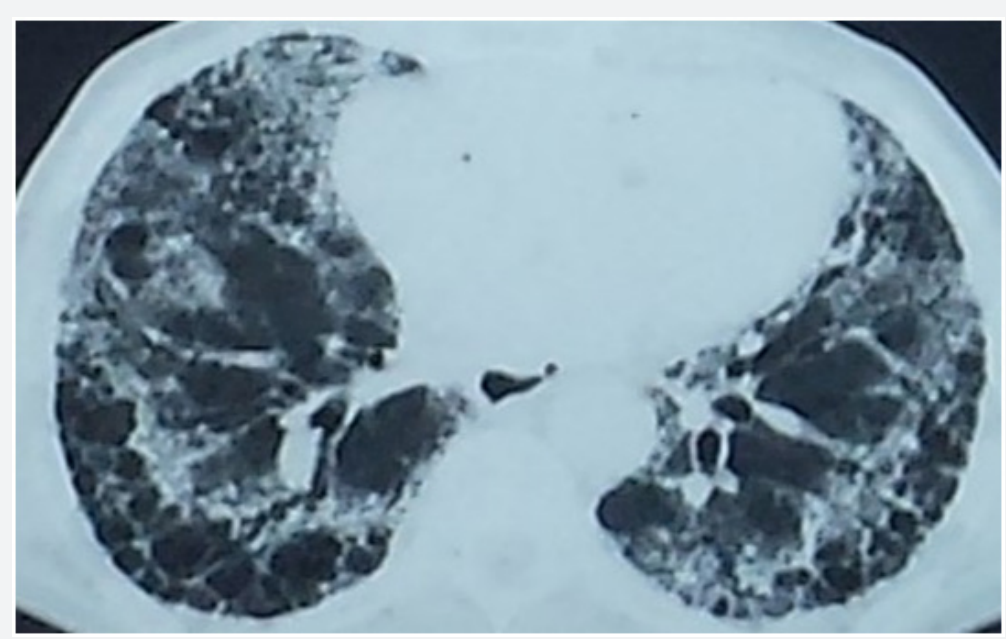

Figure 3: Fibrosing interstitial pneumonitis with interstitial anomalies in the form of numerous inter and intra-lobular pleural cross-links in the middle and lower right lobes.



Figure 4: Micronodules, confluent acuminate nodules, excavated condensations and unilateral cavities. 


\section{Discussion}

TB is still one of the most common infectious diseases in the world. It is the second leading cause of death due to infectious disease worldwide [4-6], especially in the developing world, after HIV [5-8]. In Mauritania, the limited diagnostic capacity of TB remains a challenge to improve case detection rates. The clinical diagnosis of this serious disease is difficult. Laboratory diagnosis (sputum examination) and chest X-ray are often required to establish the diagnosis. The demonstration of a radiological parenchymal infiltrate is a strong argument in its favor. Early diagnosis of pulmonary TB is not always easy to establish since very often it shares non-specific radiographic changes with the more common pathological pneumopathies, leading to late diagnosis [9]. In our study, the average time to diagnosis was 7 weeks, with chest pain, chronic cough, fever, and dyspnea as the most common signs and symptoms. The time to diagnosis and presenting signs and symptoms were similar to those reported by other African authors [7-9]. Initial chest X-rays performed in our patients were normal. Other authors have observed that chest X-ray may be normal in 15\% of smear-positive TB patients [10]. Radiologically, pulmonary TB is typically characterized by parenchymal, ganglionic, pleural or rarely diffuse hematogenous pulmonary involvement [11]. Although microscopic examination of sputum is commonly practiced, in many developing countries, chest X-ray remains a standard diagnostic tool for pulmonary TB. However, chest X-ray is inadequate in 30 to $40 \%$ of cases [12]. Chest X-ray has low sensitivity and low specificity and may show images of parenchymal abnormalities with delay.

Our study provides the first CT data from patients with smear-negative pulmonary TB in Mauritania. Indeed, the role of radiographic imaging in the diagnosis and monitoring of pulmonary TB is crucial. Most often, the aspects and morphology seen in radiographic images of lesions are suggestive of TB. However, in "radiographically silent forms," CT can provide images of thin sections in high resolution and reveal 'cryptic' TB. In restricted circumstances, CT becomes an essential diagnostic tool to identify radiographically negative patients with active TB [13]. An early performance of a chest CT scan can have an important impact on case management in such cases $[12,13]$. However, due to the high cost of purchase and maintenance of CT scan, especially in a country where the incidence of TB remains high, it is essential to determine the most appropriate place of CT in establishing the diagnosis of pulmonary TB. The present prospective study performed over 12 months was limited to 24 radiographically negative patients with confirmed pulmonary TB who benefited from CT scan. The mean age, sex ratio, and origin of our patients were comparable to those of a previous study conducted in the same region in Mauritania [7].

CT has become an essential complementary examination in the exploration of thoracic and especially parenchymal pathologies. In our case series, CT abnormalities have been dominated by images of lobar and micronodular alveolar condensation. CT is more sensitive to detect micronodules, determine their random diffuse distribution at the pulmonary level, and detect small lesions which may be located in areas that are poorly explored in standard radiography, such as the pulmonary apex (area of particular interest in the study of tuberculosis) and also subpleural, juxta-mediastinal and juxta-diaphragmatic regions. CT thus makes it possible to evaluate the signs of activity of the disease, to better detect the evolution of complications and sequelae, to guide the location of biopsy samples in atypical forms, making it possible to avoid thoracotomies, and to follow the evolution of lesions under treatment [12].

However, CT scan has several limitations which are mainly represented by its radiating nature, the need in some cases for an injection of ioversol contrast medium, and the need for cooperation of the patient who must remain immobile and in apnea during imaging, which is difficult to attain in children and agitated patients. These limitations may sometimes cause the examination to be deferred or substituted by another technique $[14,15]$. One of the major limitations of CT is the examination of the mediastinum. Indeed, its analysis requires an injection of contrast medium and a higher irradiation dose because the spontaneous contrast of the mediastinal structures is weak. Thus, the irradiation of an examination of the mediastinum cannot be reduced as much as that of an image acquisition of the pulmonary parenchyma. As a result, the scanner is too small to be currently adapted to the research and analysis of mediastinal pathologies, even if it allows a rough visualization of the main anatomical structures. A second major limitation of the dose scanner is related to the morphotype of the patient. The more corpulent a patient is, the more X-rays will be absorbed, and the poorer will be the quality of the image. Thus, beyond a certain weight, the poor image quality may compromise the establishment of the correct diagnosis. Another limitation of CT is the difficulties in the analysis of interstitial parenchymal abnormalities. Indeed, the CT abnormalities encountered in diffuse interstitial pneumonitis are subtle, especially in the initial phase when the main lesions are sometimes not very distinct [14]. Despite these limitations, CT imaging may be helpful in certain circumstances to diagnose TB.

\section{Conclusion}

In Mauritania, the prevalence of TB is still high, and the diagnosis of $\mathrm{TB}$ with negative bacteriological examination remains difficult because of the insufficiency of the technical platform and limited financial resources of patients. From a pragmatic perspective, the diagnostic approach usually consists of clinical examination and chest X-ray. However, for diagnostic confirmation, bacteriological and/or histological examinations are required. Although the standard chest X-ray remains the reference diagnostic method in most cases, CT may be crucial in some cases for the diagnosis and monitoring of pulmonary TB. 


\section{Declaration of Transparency}

The authors have no conflict of interest to declare.

\section{Contributions of the Authors}

Boushab Mohamed Boushab (bboushab@gmail.com) :1st author and Corresponding author, he has been involved in drafting the manuscript, made substantial contributions to study conception and design, clinical data verification, discussion section.

Sidi Yeslem Ould-Bahiya, (souldhamdi@yahoo.fr): 2nd author, manuscript correction.

Mamoudou Savadogo (savadoma@gmail.com): 3rd author, manuscript correction.

Abdoulaye Mamadou Traore (amtraore2008@gmail.com): 4th author, manuscript correction.

Leonardo Kishi Basco (lkbasco@yahoo.fr): 5th author, manuscript correction.

All authors have read and approved the manuscript

\section{Acknowledgments}

We are grateful for the tireless efforts of the medical staff for their excellent patient care at Kiffa Regional hospital.

\section{References}

1. World Health Organization (2015) Global tuberculosis report 2014. WHO, Geneva.

2. Piatek AS, Van Cleeff M, Alexander H, Coggin WL, Rehr M, et al. (2013) GeneXpert for TB diagnosis: planned and purposeful implementation. Glob Health Sci Pract 1(1): 18-23.

3. Perkins, Roscigno G, Zumla A (2006) Progress towards improved tuberculosis diagnostics for developing countries. Lancet 367: 942943

4. Martínez Romero A, Ortega Sanchez JL, González Yañez MG, Hernandez Salgado JR, Ruiz Baca E, et al. (2012) Comparison of acid-fast bacilli smear and chromatographic immunoassay in patients with active tuberculosis. Afr J Microbiol Res 6(8): 1690-1693.

5. Campos LC, Rocha MVV, Willers DMC, Silva DR (2016) Characteristics of patients with smear-negative pulmonary tuberculosis (TB) in a region with high TB and HIV prevalence. PLoS One 11(1): e0147933.

6. Cavanaugh JS, Shah NS, Cain KP, Winston CA (2012) Survival among patients with HIV infection and smear-negative pulmonary tuberculosis - United States, 1993-2006. PLoS One 7(10): e47855.

7. Boushab BM, Savadogo M, Sow MS (2016) Prévalence de la tuberculose pulmonaire à bacilloscopie positive dans un centre hospitalier d'Aïoun (Hodh El Garbi). Rev Pneumol Clin 72: 243-247.

8. Affolabi D, Akpona R, Odoun M, Alidjinou K, Wachinou P, et al. (2011) Smear-negative, culture-positive pulmonary tuberculosis among patients with chronic cough in Cotonou, Benin. Int J Tuberc Lung Dis 15(1): $67-70$

9. Harries AD, Hargreaves NJ, Kwanjana JH, Salaniponi FM (2001) Clinical diagnosis of smear-negative pulmonary tuberculosis: an audit of diagnostic practice in hospitals in Malawi. Int J Tuberc Lung Dis 5(12): 1143-1147.

10. Hawken MP, Muhindi DW, Chakaya JM, Bhatt SM, Ng'ang'a LW, et al. (2001) Under-diagnosis of smear-positive pulmonary tuberculosis in Nairobi, Kenya. Int J Tuberc Lung Dis 5(4): 360-363.

11. Biadglegne F, Rodloff AC, Sack U (2014) A first insight into high prevalence of undiagnosed smear-negative pulmonary tuberculosis in northern Ethiopian prisons: Implications for Greater Investment and Quality Control. PLoS One 9(9): e106869.

12. Debray MP (2005) Imagerie thoracique, techniques et indications, Encycl Méd Chir, Médecine 2(1): 42-55.

13. Claessens YE, Debray MP, Tubach F, Brun AL, Rammaert B, et al. (2015) Early chest computed tomography scan to assist diagnosis and guide treatment decision for suspected community-acquired pneumonia. Am J Respir Crit Care Med 192(8): 974-982.

14. Ohanaa M, Ludesa BC, Schaalc M, Meyera E, Jeunga MY, et al. (2017) What future for chest x-ray against ultra-low-dose computed tomography? Rev Pneumol Clin 73(1): 3-12.

15. Lee SW, Kim Y, SS Shim, Lee JK, Lee SJ, et al. (2014) Image quality assessment of ultra-low-dose CT chest using sinogram-affirmed iterative reconstruction. Eur Radiol 24(4): 817-826.

\section{Your next submission with Juniper Publishers} will reach you the below assets

- Quality Editorial service

- Swift Peer Review

- Reprints availability

- E-prints Service

- Manuscript Podcast for convenient understanding

- Global attainment for your research

- Manuscript accessibility in different formats (Pdf, E-pub, Full Text, Audio)

- Unceasing customer service

Track the below URL for one-step submission https://juniperpublishers.com/online-submission.php 